\title{
Successive Interference Cancellation in Clipped and Product Combining aided FFH Multi-User Systems
}

\author{
Sohail Ahmed, Lie-Liang Yang and Lajos Hanzo \\ School of ECS, University of Southampton, SO17 1BJ, UK. \\ Tel: +44-23-8059 3364, Fax: +44-23-8059 4508 \\ Email: \{sa03r,lly,lh\}@ecs.soton.ac.uk; http://www-mobile.ecs.soton.ac.uk
}

\begin{abstract}
In this contribution, we propose two successive interference cancellation (SIC) schemes for a fast frequency hopping (FFH) multiple access (MA) system using $M$-ary frequency shift keying (MFSK) and invoking multi-user detection (MUD). One of the proposed schemes invokes clipped combining, while the other scheme employs both product combining and clipped combining. The SIC schemes are adapted from a scheme proposed by U.-C. Fiebig in 1996. The basic principle of the SIC schemes is that detection is carried out in multiple stages and during each stage, only the most reliable symbols are detected. In subsequent stages, the interference contributed by the already detected symbols may be removed. The performance of the proposed schemes is evaluated and compared to that of Fiebig's scheme, when the FFH-MFSK system operates in a Nakagami$m$ fading MA channel. The simulation results demonstrate that the proposed schemes attain a better bit error rate performance than Fiebig's scheme.
\end{abstract}

\section{INTRODUCTION}

In a conventional cellular system, the base station (BS) receiver usually employs a separate receiver corresponding to each user, each of which is the same as that used in single user detection (SUD). Given the knowledge of all users' addresses, the BS receiver may invoke Multi-User Detection (MUD), which jointly detects all users' signals. Specifically, when multi-stage successive interference cancellation (SIC) assisted MUD is employed, the symbols detected in a specific stage can be removed in the subsequent stages, thereby reducing the interference constituted by these already detected users. In the context of FFH-MA systems using MFSK, various SIC techniques have been proposed in the open literature for mitigating the multi-user interference (MUI) [1]-[5]. All these schemes are applicable to FFH-MFSK employing conventional hard limiting majority vote (HLMV) based combining [1], [3]. Motivated by the promising performance gains that some of the combining schemes yield compared to HLMV combining [6], [7], in this contribution, we propose two novel SIC methods, one of which invokes the clipped combining while the other employs both clipped combining and product combining. These SIC schemes are based on modifications of Fiebig's method [3], which was proposed for FFH-MFSK system employing HLMV combining. It consisted of multiple SIC stages and at each stage the interference originally imposed by the symbols that were successfully detected in the

The financial support of the EPSRC, UK and EU under the auspices of the Phoenix and Newcom projects and of Higher Education Commission, Pakistan is gratefully acknowledged. previous stage were cancelled.

The rest of this paper is structured as follows. In Section II, the system under consideration is briefly described and in Section III we highlight the proposed SIC schemes. In Section IV, we will demonstrate with the aid of simulation results that the proposed SIC methods result in useful performance gains and outperform Fiebig's scheme [3]. Finally, in Section IV, we present our conclusions.

\section{SYSTEM DESCRIPTION}

The FFH-MFSK system considered consists of a single cell serving multiple users with the aid of a BS and utilizing a spread spectrum bandwidth $W_{s s}$, as in [3]. The $k$ th user, $k=1,2, \ldots, U$, is assigned a unique and random $L$-tuple FH address denoted by $\mathbf{a}_{\mathbf{k}}=\left[a_{k}(0), a_{k}(1), \ldots, a_{k}(L-1)\right]$, which is output by the PN generator, where $a_{k}(l) \in \mathrm{GF}(M)$, $l=0,1, \ldots, L-1, L$ is the number of frequency hops per symbol and $\mathrm{GF}(M)$ represents a Galois field having $M$ elements. The frequency of the the $k$ th user's hopped signal in the $l$ th hop may assume any of the $M$ legitimate values and is chosen according to

$$
\mathcal{S}_{\mathbf{k}}=\left[\mathcal{S}_{k}(0), \mathcal{S}_{k}(1), \ldots, \mathcal{S}_{k}(L-1)\right]=X_{k} . \mathbf{1} \oplus \mathbf{a}_{\mathbf{k}},
$$

where 1 denotes a unit vector of length $L, X_{k}$ is the symbol to be transmitted by the $k$ th user and $\oplus$ denotes addition in the Galois field. The components of $\mathcal{S}_{k}$ are passed to a MFSK modulator, where a MFSK tone is chosen for transmission which is determined by the value of $\mathcal{S}_{k}(l)$ during the lth hop of duration $T_{h}$. Since each symbol consists of $L$ hops, the hop or chip interval $T_{h}$ is related to the symbol interval $T_{s}$ by the relation $T_{h}=T_{s} / L$. We assume that the bandwidth occupied by the signal transmitted during each FFH chip interval is given by $R_{h}=1 / T_{h}$. The frequency separation between adjacent FH tones is also assumed to be $R_{h}$. Thus, the orthogonality of the FFH frequencies is maintained.

The channel is modeled by Nakagami- $m$ fading for each of the transmitted frequencies. We assume that the frequency separation between the adjacent signalling frequencies is higher than the coherence bandwidth of the channel and thus, each signalling tone can be assumed to experience independent fading. Moreover, the fading envelopes experienced by the various users are also independent of each other. Furthermore, the transmitted signals are corrupted by Additive White Gaussian Noise (AWGN) of a one-sided power spectral density $N_{0}$. 


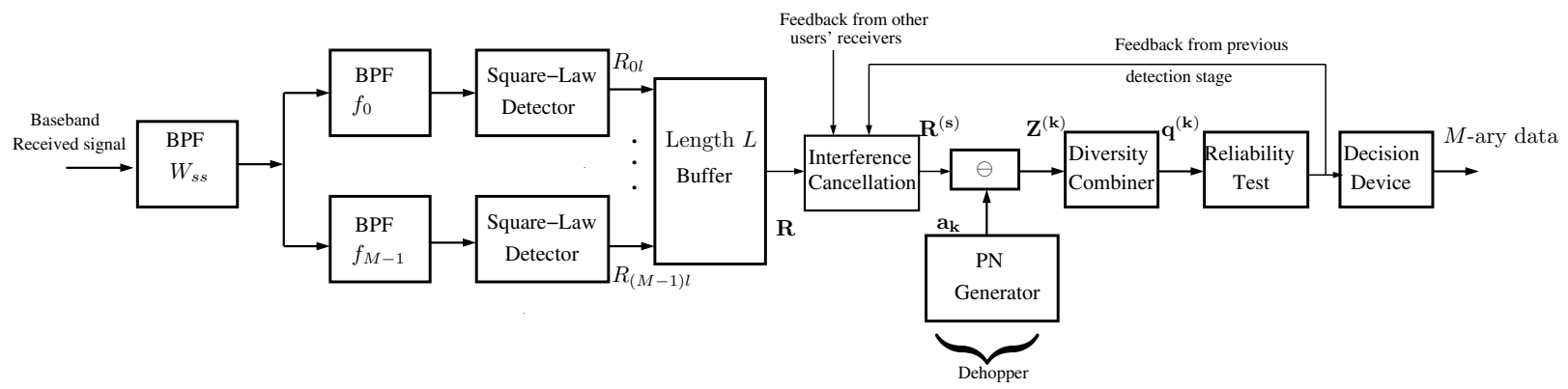

Fig. 1. Schematic of the FFH-MFSK receiver of the $k$ th user, employing SIC assisted MUD

We assume that the system supports $U$ simultaneous users. When a particular user transmits a signal to the BS, the signals transmitted by all other users may be modeled as continuouswave interfering tones. We assume a chip-synchronous system, where the chip transitions of all users are aligned. Furthermore, perfect power control is assumed, hence the power of all the signals received by the BS from all users is identical.

The BS receiver schematic is shown in Fig. 1. After carrier demodulation, a bank of $M$ square-law detectors detect the energy received in each MFSK tone. Then SIC is employed for cancelling MUI, which is discussed in the context of the proposed SIC schemes in the forthcoming section.

\section{Proposed SIC SCHEMES}

In this section, we propose two SIC schemes that are adapted from Fiebig' s scheme [3]. However, instead of using HLMV combining as in [3], the first SIC scheme employs the clipped combining and the second SIC scheme employs both the product combining and the clipped combining [6]. In the context of the proposed SIC schemes, the detection process consists of a conventional detection (CD) stage and multiple SIC stages. In each stage, only the most reliable symbols are detected. To determine the reliability of a symbol, Viterbi's Ratio Threshold Test (RTT) [8] is proposed, which will be discussed below. After the most reliable symbols have been successfully demodulated at the output of an SIC stage, the received time-frequency matrix $\mathbf{R}$, shown in Fig. 1, is modified by erasing the signal contributions of the already detected symbols. Therefore, due to these interference cancellation steps, the number of interferers in the next stage of detection is reduced, facilitating more confident demodulation of the hitherto undetected symbols.

\section{A. Clipped Combining Aided SIC}

The first proposed SIC scheme employs clipped combining [6], which is also referred to as soft limiting combining (SLC) [7]. Therefore, we refer to this scheme as the SLC-SIC scheme. It is discussed below with the aid of Fig. 2. Note that we have replaced operations in the Galois field with Modulo$M$ operations, for ease of analysis.

1) As seen in Fig. 1, the received signal recorded at the output of the energy detectors corresponding to the $M$ frequency tones and $L$ time slots can be visualized as a $(M \times L)$-dimension time-frequency matrix $\mathbf{R}$, in which the element $R_{m l}, m=0,1, \ldots, M-1, l=$ $0,1, \ldots, L-1$, represents the superposition of the signal energy transmitted by up to $U$ users and the AWGN received in the $m$ th tone during the $l$ th hop. As seen in Figs. 1 and 2, $\mathbf{R}$ is separately dehopped by each receiver using the corresponding unique address, resulting in the matrix $\mathbf{Z}^{(k)}, k=1,2, \ldots, U$, for the $k$ th user. The dehopping process of user $k$ may be expressed as

$$
\begin{aligned}
& Z_{n l}^{(k)}=R_{m l}, \text { given } n=m \ominus a_{k}(l), \\
& m, n=0,1, \ldots, M-1 ; l=0,1, \ldots, L-1 .
\end{aligned}
$$

2) Clipped combining is performed on the dehopped signals, resulting in $M$ decision variables for each of the users, as seen in Fig. 1. The process of clipping can be expressed as [6]

$$
f\left(Z_{m l}^{(k)}\right)= \begin{cases}C, & \text { if } Z_{m l}^{(k)} \geq C \\ Z_{m l}^{(k)}, & \text { otherwise }\end{cases}
$$

where $C$ represents an appropriately chosen clipping threshold. The decision variable recorded after clipped combining for the $k$ th user is given by

$$
q_{m}^{(k)}=\sum_{l=0}^{L-1} f\left(Z_{m l}^{(k)}\right), \quad m=0,1, \ldots, M-1 .
$$

3) The following test, which we refer to as the RTT [8], is performed for each of the users, as shown in Fig. 1. Let $Y_{1}^{(k)}$ be the largest and $Y_{2}^{(k)}$ be the second largest of all the $M$ decision variables $\left\{q_{m}^{(k)}\right\}$ corresponding to the $k$ th user. Then we define the ratio

$$
\lambda^{(k)}=\frac{Y_{1}^{(k)}}{Y_{2}^{(k)}} .
$$

Now, if we have $\lambda^{(k)} \geq \lambda_{T}^{(c c)}$, where $\lambda_{T}^{(c c)}$ is an appropriately chosen threshold in the context of clipped combining, then we establish that the symbol is reliable. The reliable symbols are demodulated in the conventional manner. That is, if the $j$ th user's symbol, $j=1,2, \ldots, U$ satisfies the above-mentioned RTT, then $X_{j}^{(d)}$ is the estimate of the $j$ th user's symbol, where $X_{j}^{(d)}=0,1, \ldots, M-1$ is the index of $Y_{1}^{(j)}$. If all users' symbols pass the test, the detection process for 
the symbol considered is deemed complete. On the other hand, if a user's symbol fails to satisfy the RTT, its detection is deferred until later stages. If no user passes the test, we choose only the $p$ th user's symbol for demodulation and subsequent erasure, such that

$$
\lambda^{(p)}=\max \left[\lambda^{(1)}, \lambda^{(2)}, \ldots, \lambda^{(U)}\right], \quad p=1,2, \ldots U .
$$

4) If some but not all symbols are reliably detected, proceed with the $s$ th stage of SIC, where $s=1,2, \ldots$ and we use $s=0$ to indicate the CD stage, which consists of the Steps 1 to 3 above. We also define $\mathbf{R}^{(s)}$ as the modified form of $\mathbf{R}$ at the $s$ th stage, as seen in Fig. 1. By this definition, we have $\mathbf{R}=\mathbf{R}^{(0)}$. The $s$ th stage of the SIC, $s \geq 1$, consists of the following steps:

a) Modify matrix $\mathbf{R}^{(s-1)}$ by erasing the signals which correspond to all the symbols that were reliably detected in the $(s-1)$ th stage (step 3$)$. Hence, if $X_{k}^{(d)}$ is the detected symbol for the $k$ th user, then, after the interference cancellation, we have

$$
R_{m l}^{(s)}= \begin{cases}0 & \text { for } m=X_{k}^{(d)} \oplus a_{k}(l) \\ R_{m l}^{(s-1)} & \text { otherwise }\end{cases}
$$

b) Repeat Steps 1 to 3 outlined above applicable to the matrix $\mathbf{R}^{(s)}$, for all users whose symbols have not yet been detected, resulting in successful detection of the reliable sysmbols that satisfy the RTT.

The SIC operations described above can be continued until all users' symbols meet the RTT condition or, until a pre-defined number of stages $S$ have been completed. In the latter case, all remaining symbols in the last stage are detected using the conventional approach, without invoking the RTT. In Fig. 2, we assume that symbol $X_{B}=4$ of user B satisfies the RTT, which is hence demodulated and erased from the matrix $\mathbf{R}$ to obtain the modified matrix $\mathbf{R}^{(1)}$ shown in Fig. 2(c). Based on $\mathbf{R}^{(1)}$, the symbols of both user $\mathrm{A}$ and $\mathrm{C}$ can be reliably detected in the first SIC stage $(s=1)$.

\section{B. Product and Clipped Combining Aided SIC}

The second SIC scheme that we propose, referred to as the PC-SLC-SIC scheme, employs both the product combining [6] and the clipped combining schemes. Specifically, in the CD stage, product combining is employed, while in the subsequent stages of SIC, clipped combining is used. The PC-SLC-SIC scheme can be described by these steps:

1) The received signals are separately dehopped by each receiver using the corresponding unique address, resulting in matrix $\mathbf{Z}^{(k)}$ for the $k$ th user, as defined by (2).

2) Product combining is performed on the dehopped signals, resulting in $M$ decision variables for each of the user, as given by [6]

$$
q_{m}^{(k)}=\prod_{l=0}^{L-1} Z_{m l}^{(k)}, \quad m=0,1, \ldots, M-1 .
$$

3) The RTT statistics $\lambda^{(k)}$ are computed according to (5). Now, if we have $\lambda^{(k)} \geq \lambda_{T}^{(p c)}$, where $\lambda_{T}^{(p c)}$ is an appropriately chosen threshold in the context of product combining, then we establish that the symbol is reliable. The reliable symbols are demodulated as shown in Section III-A (Step 3). If all users' symbols pass the RTT, the detection of the symbol considered is complete. If a user's symbol fails the RTT, its detection is deferred until later SIC stages. If no user passes the test, we choose only the user with the highest $\lambda$ value for demodulation and subsequent erasure, as defined by (6).

4) If some but not all symbols are reliably detected, proceed with the $s$ th stage of SIC, where $s=1,2, \ldots$ and $\mathbf{R}^{(\mathbf{s})}$ is defined as in Section III-A. The sth stage, $s \geq 1$, consists of the following steps:

a) Modify $\mathbf{R}^{(s-1)}$ by cancelling the signals which correspond to all the symbols that were reliably detected in step 3, as highlighted by (7).

b) Repeat step 1 to 3 of Section III-A applicable to the matrix $\mathbf{R}^{(s)}$ for all users whose symbols have not yet been detected, resulting in successful detection of the reliable sysmbols that satisfy the RTT. Note that for all $s>0$, clipped combining, as characterised in (3) and (4), is invoked.

As in the case of SLC-SIC, the SIC stages in the PC-SLCSIC scheme can be extended, until all symbols are reliably detected or until we reach $s=S$.

\section{Simulation Results}

In this section we present our BER performance results for the SIC schemes proposed in Section III and for Fiebig's scheme [3], when they are employed in FFH-MFSK system operating in a frequency-flat Nakagami- $m$ fading MA channel. For each SIC scheme under consideration, following the CD stage, a maximum of three SIC stages per symbol have been allowed, after which the detection process is concluded, i.e. we have $S=3$. Additionally, the optimum clipping thresholds $C$ and the RTT thresholds $\lambda_{T}^{(c c)}$ and $\lambda_{T}^{(p c)}$, as defined in Section III, have been employed in all simulations.

In Fig. 3, we have plotted the BER achievable by the SLCSIC, the PC-SLC-SIC and Fiebig's scheme [3], against the number of active users $U$. The corresponding BER results achievable using the HLMV combining and the SLC in CD mode, i.e. without SIC, have also been shown in Fig. 3, where $E_{b} / N_{0}=25 \mathrm{~dB}$, the fading parameter was $m=1$ implying Rayleigh fading and $M=128$ and $L=7$ were assumed. As seen in Fig. 3, all SIC schemes outperform the $\mathrm{CD}$ schemes as expected. Moreover, the SLC-SIC and the PCSLC-SIC schemes outperform Fiebig's scheme [3], when we have $U<45$. For a high number of users, Fiebig's scheme [3] performs better than the other two SIC schemes, although for $U \geq 60$ the BER achieved by all the SIC schemes considered is excessive for practical use. We also observe from Fig. 3 that the performance of the PC-SLC-SIC scheme is poorer than those of the other two SIC schemes, when $U$ is high. However, when we have $U<45$, the PC-SLC-SIC scheme outperforms the other two schemes.

In Fig. 4, the same comparisons have been carried out as those shown in Fig. 3, but assuming $E_{b} / N_{0}=15 d B$. As seen 


\begin{tabular}{|c|c|l|l|l|l|l|l|}
\hline$U$ & $E_{b} / N_{0}$ & \multicolumn{2}{|c|}{ Fiebig's scheme } & \multicolumn{2}{c|}{ SLC-SIC } & \multicolumn{2}{c|}{ PC-SLC-SIC } \\
\hline & $(\mathrm{dB})$ & BER & $S_{a v}$ & BER & $S_{a v}$ & BER & $S_{a v}$ \\
\hline 20 & 25 & $2.40882 \mathrm{e}-5$ & 1.656 & $2.63559 \mathrm{e}-6$ & 0.5946 & $2.12913 \mathrm{e}-6$ & 1.0342 \\
20 & 15 & 0.00332699 & 2.947 & 0.000296844 & 1.941 & 0.000523414 & 1.936 \\
40 & 25 & 0.001005 & 2.70 & 0.000841 & 1.064 & 0.00057852 & 2.334 \\
40 & 15 & 0.0265562 & 2.955 & 0.0107515 & 2.635 & 0.0161537 & 2.716 \\
60 & 25 & 0.010733 & 2.9856 & 0.0174295 & 1.989 & 0.0280861 & 2.94 \\
60 & 15 & 0.102039 & 2.831 & 0.0862992 & 2.607 & 0.113554 & 2.954 \\
\hline
\end{tabular}

TABLE I

THE ACHIEVABLE BER AND THE AVERAGE NUMBER OF SIC STAGES REQUIRED BY THE FFH-MFSK MA RECEIVER USING VARIOUS SIC SCHEMES FOR COMMUNICATING OVER A RAYLEIGH FADING CHANNEL, ASSUMING $L=7$ AND $M=128$

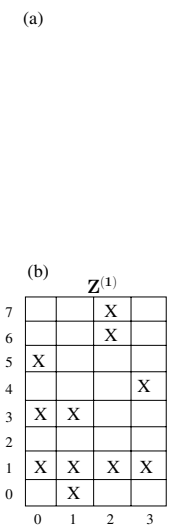

(c)
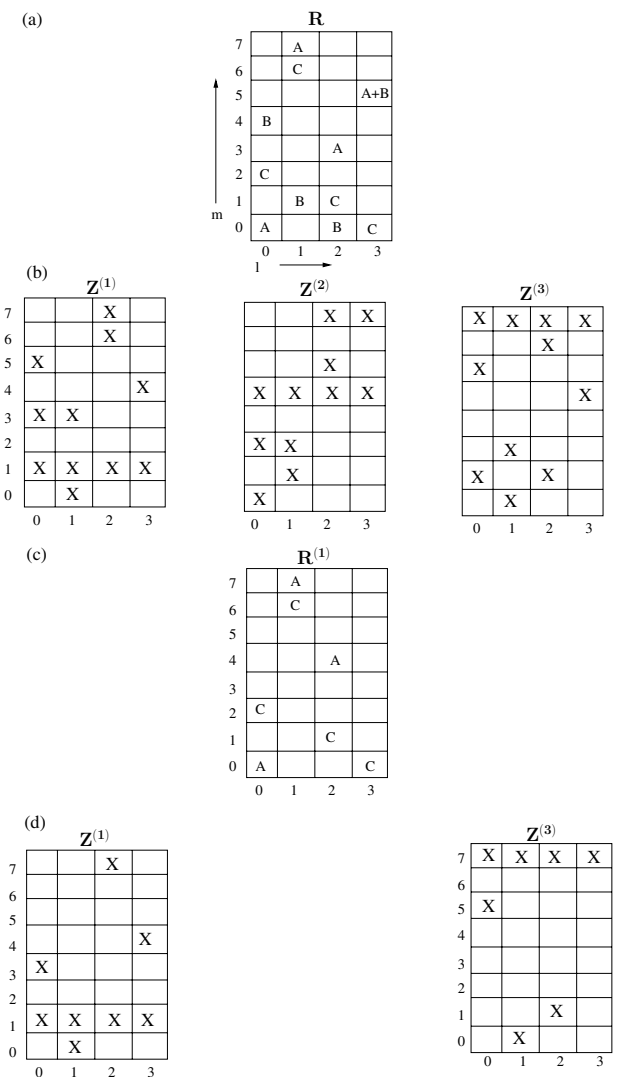

Fig. 2. An example of SIC in the context of an 8-ary system having $L=4$ and $U=3$. The three users are assumed to transmit symbols 1 , 4 and 7 and have the addresses [7,6,2,4], [0,5,4,1] and [3,7,1,2], respectively. Blank elements of all the matrices indicate the presence of AWGN only. (a) Received $M \times L$ matrix $\mathbf{R}$. The symbols transmitted by the three users, generated using (1), are denoted by the letters A, B and C, respectively. (b) Dehopped matrices $\mathbf{Z}^{(\mathbf{k})} k=1,2,3$ corresponding to the three users, generated using (2). An $\mathrm{X}$ in an element denotes presence of transmitted signal in the corresponding tone and hop. (c) Modified received matrix $\mathbf{R}^{(\mathbf{1})}$, generated using (7), after cancellation of all entries owing to symbol 4 of user B, which is assumed to have been successfully detected after diversity combining and invoking the RTT. (d) Dehopped matrices of users A and C in the first stage $(s=1)$ of SIC generated by invoking (2) on $\mathbf{R}^{(\mathbf{1})}$.

in Fig. 4, when the SNR is relatively low, the performance of Fiebig's scheme [3] degrades more dramatically compared to those of the other two SIC schemes. By comparing the results shown in Figs. 3 and 4, we may conclude that Fiebig's scheme [3] is more sensitive to noise and yields a good performance only when the SNR is sufficiently high. Furthermore, in Fig. 4, the performance of the SLC-SIC is visibly better than

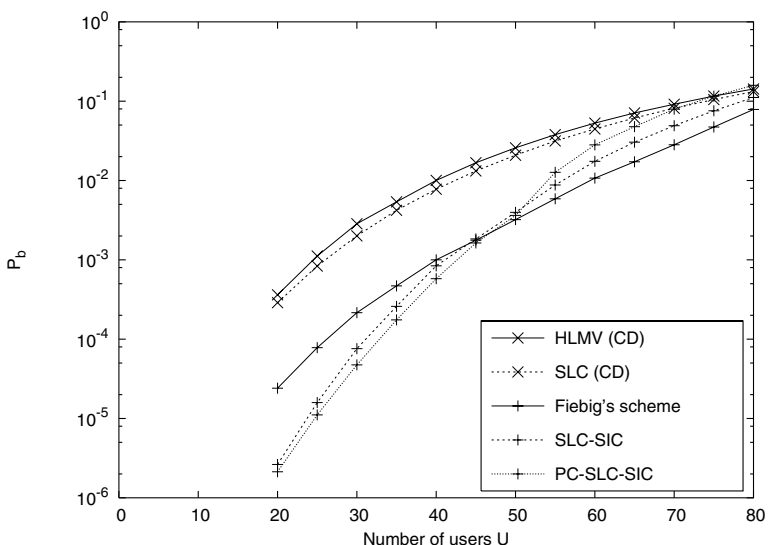

Fig. 3. BER versus number of users performance for various $\mathrm{CD}$ and SIC schemes employed in FFH-MFSK communicating over a Nakagami- $m$ fading channel, assuming $L=7, M=128, m=1$ and $\mathbf{E}_{\mathbf{b}} / \mathbf{N}_{\mathbf{0}}=\mathbf{2 5 d B}$.

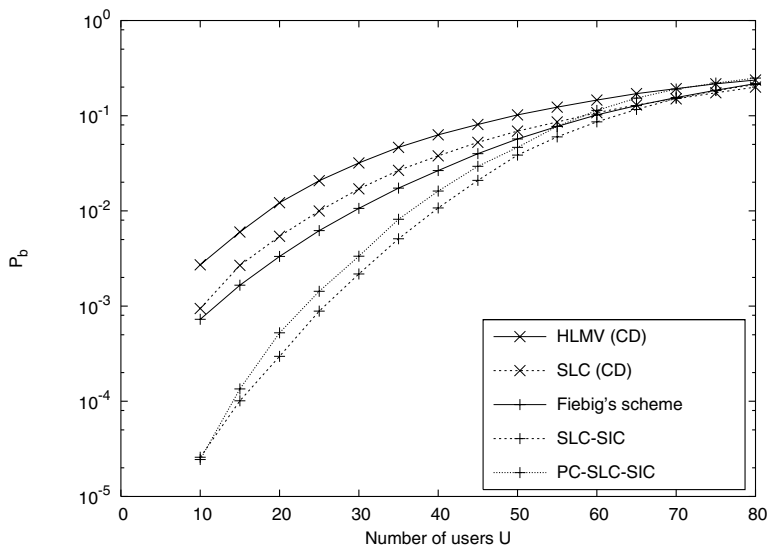

Fig. 4. BER versus number of users performance for various CD and SIC schemes, employed in FFH-MFSK communicating over a Nakagami- $m$ fading channel, assuming $L=7, M=128, m=1$ and $\mathbf{E}_{\mathbf{b}} / \mathbf{N}_{\mathbf{0}}=\mathbf{1 5 d B}$.

that of the PC-SLC-SIC for most values of $U$. The comparison of the results plotted in Figs. 3 and 4 also shows that the PCSLC-SIC scheme is more sensitive to the SNR encountered than the SLC-SIC scheme.

The BER performance of the schemes under consideration has been portrayed for $m=1.5$ in Fig. 5 and for $m=10$ in Fig. 6 . In both these figures, $E_{b} / N_{0}=15 d B$ has been assumed and the remaining parameters are the same as those assumed in Fig. 4. Since increased value of $m$ implies less severe fading in the context of the Nakagami model, as expected the performance of all the SIC and CD schemes improved, 
as seen by comparing Figs. 5 and 6 with Fig. 4. We also observe from these two figures that the PC-SLC-SIC scheme significantly benefits from a reduced fading intensity. Thus, if we compare the results of Figs. 5 and 6 with those shown in Fig. 4, where the same SNR value is assumed, we observe that when $m=1.5$ is assumed in Fig. 5, the PC-SLC-SIC scheme outperforms the SLC-SIC scheme for low values of $U$. Furthermore, when we have $m=10$ in Fig. 6, the PCSLC-SIC scheme yields a better BER performance than the other two SIC methods considered, almost regardless of the value of $U$. This observation can be explained by considering the multiplication operation involved in product combining; under severe fading conditions, the product of energy detector outputs may become very low, thus resulting in an increased probability of detection errors.

Another factor that governs the performance of the SIC

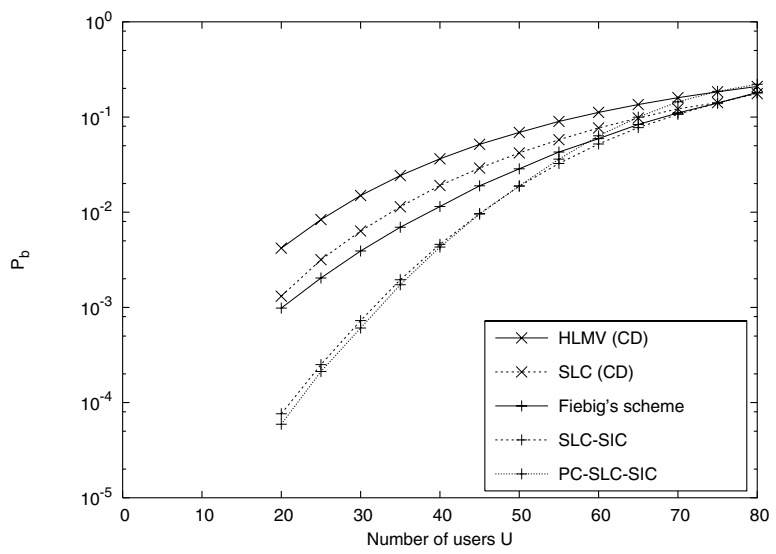

Fig. 5. BER versus number of users performance for various $\mathrm{CD}$ and SIC schemes employed in FFH-MFSK communicating over Nakagami- $m$ fading channel, assuming $L=7, M=128, \mathbf{m}=\mathbf{1 . 5}$ and $E_{b} / N_{0}=15 \mathrm{~dB}$.

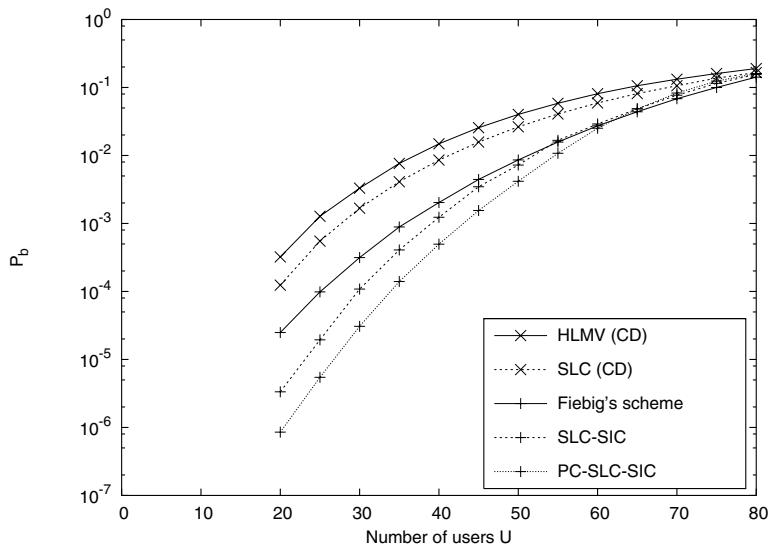

Fig. 6. BER versus number of users performance for various $\mathrm{CD}$ and SIC schemes employed in FFH-MFSK communicating over a Nakagami- $m$ fading channel, assuming $L=7, M=128, \mathbf{m}=\mathbf{1 0}$ and $E_{b} / N_{0}=15 \mathrm{~dB}$.

schemes is the number of SIC stages required for achieving the lowest possible BER at a given set of system parameters. In Table I, some values of the lowest possible BER achievable using the SIC schemes considered have been listed. The parameter $S_{a v}$ denotes the average number of SIC stages needed to achieve the lowest BER. The results listed in Table I demonstrate that the SLC-SIC scheme is also attractive from the perspective of reduced complexity, since it achieves the lowest possible BER at a lower value of $S_{a v}$. Similarly, the performance of the PC-SLC-SIC scheme is inferior in comparison to that of the SLC-SIC scheme, but better than that of Fiebig's scheme [3].

\section{CONCLUSION}

We have proposed two novel SIC schemes for a FFH-MA system using MFSK that were derived from Fiebig's SIC scheme [3]. However, whereas Fiebig's scheme and all other SIC schemes designed for FFH-MA are applicable to HLMV combining [1]-[5], one of our proposed schemes invokes the SLC, while the other uses product combining in the CD stage and SLC in the SIC stages. We compared the BER performance of the proposed SIC schemes with that of Fiebig's scheme [3], when the system communicates over a Nakagami$m$ fading MA channel. Our simulation results given in Figs. 3 to 6 and listed in Table I show that the SLC-SIC and the PC-SLC-SIC schemes attain better performance than Fiebig's scheme [3] for most practical values of the system parameters. Moreover, the BER performance of Fiebig's scheme [3] is more adversely affected than that of our proposed schemes, when the SNR is not sufficiently high. When the SNR is low, the SLC-SIC yields a better performance than the other two schemes. The PC-SLC-SIC scheme is the better design option, when the number of active users is low, provided that the SNR value is high enough or the fading is less severe. Both the proposed SIC schemes are also attractive in terms of attaining the lowest possible BER using low number of SIC stages. A specific drawback of the proposed schemes is that their achievable performance is dependent upon the choice of the RTT thresholds that determine which symbols are reliable. We conclude that for most values of system parameters that are of practical interest, our SIC schemes proposed in Section III constitute better design options than Fiebig's scheme [3].

\section{REFERENCES}

[1] U. Timor, "Multi-stage decoding of frequency-hopped multilevel FSK system,” Bell System Technical Journal, vol. 60, pp. 471-483, April 1981.

[2] T. Mabuchi, R. Kohno, and H. Imai, "Multiuser detection scheme based on cancelling cochannel interference for MFSK/FH-SSMA system," IEEE Journal on Selected Areas of Communications, vol. 12, pp. 593-604, February 1995.

[3] U.-C. Fiebig, "Iterative interference cancellation for FFH/MFSK MA systems," in IEEE Proceedings of International Conference on Communications, pp. 90-95, December 1996.

[4] K. W. Halford and M. B. Pearce, "Multistage multiuser detection for FHMA," IEEE Transactions on Communications, vol. 48, pp. 1550-1562, September 2000.

[5] R. J. Kozick and B. M. Sadler, "Maximum likelihood multi-user detection fast frequency hopping/Multiple frequency shift keying systems," in IEEE Wireless Communications and Networking Conference, vol. 1, pp. 67-72, September 2000.

[6] T. A. Gulliver, E. B. Felstead, R. E. Ezers, and J. S. Wright, "A unified approach to time diversity combining of fast frequency hopped NCMFSK - anti-jam processing," in IEEE Military Communications Conference, MILCOM '94, vol. 2, pp. 415-420, October 1994.

[7] O. C. Yue, "Maximum likelihood combining for noncoherent and differentially coherent frequency hopping multiple access systems," IEEE Transactions on Information Theory, vol. IT-28, pp. 631-639, July 1982

[8] A. J. Viterbi, "A robust ratio-threshold technique to mitigate tone and partial band jamming in coded MFSK systems," in IEEE Proceedings of Military Communications Conference Record, pp. 22.4.1-22.4.5, October 1982. 